\title{
Serological survey of canine vector-borne diseases in two animal shelters in central Peninsular Malaysia
}

\author{
Vinnie-Siow, W.Y. ${ }^{1}$, Low, V.L. ${ }^{2}$, Tan, T.K. ${ }^{{ }^{*}}$, Teoh, Y.B. ${ }^{3}$, Prakash, B.K. ${ }^{4}$, Lim, Y.A.L. ${ }^{1}$ \\ ${ }^{1}$ Department of Parasitology, Faculty of Medicine, University of Malaya, Kuala Lumpur, Malaysia \\ ${ }^{2}$ Higher Institution Centre of Excellence (HICOE), Tropical Infectious Diseases Research and Education Centre (TIDREC), University of Malaya, \\ Kuala Lumpur, Malaysia \\ ${ }^{3}$ Faculty of Veterinary Medicine, Hokkaido University, Sapporo, Japan \\ ${ }^{4}$ Institute Biological Sciences, Faculty of Science, University of Malaya, Kuala Lumpur, Malaysia \\ *Corresponding authors: vanlun_low@um.edu.my (Van Lun Low); tantk@um.edu.my (Tiong Kai Tan)
}

\section{ARTICLE HISTORY}

Received: 22 July 2020

Revised: 22 December 2020

Accepted: 22 December 2020

Published: 25 March 2021

\begin{abstract}
Canine vector-borne diseases (CVBDs) are increasingly becoming a cause for global concern because of their high morbidity and mortality rates in dogs. However, information on their occurrence in Malaysia is still scanty. In this study, a total of 103 dog blood samples were collected from two animal shelters in central Peninsular Malaysia and tested for the antibodies against Ehrlichia spp., Anaplasma spp., Borrelia burgdorferi, and the antigen of Dirofilaria immitis. Of the 103 tested dogs, 44.7\% (46) were found to be seropositive for Ehrlichia spp., 30.1\% (31) for Anaplasma spp. and 13.6\% (14) for D. immitis. Co-infections of Anaplasma spp. + Ehrlichia spp. $(18.5 \%, 19)$ were most prevalent, followed by Anaplasma spp. + D. immitis (1.9\%; two) and D. immitis + Ehrlichia spp. (1.0\%; one). Furthermore, three dogs (2.9\%) were also found to have triple infection, testing seropositive for Ehrlichia spp., Anaplasma spp. and $D$. immitis. The dogs which were found to be seropositive with at least one pathogen were $66.7 \%(32 / 51)$ at shelter A, and $55.8 \%(29 / 52)$ at shelter B. Serological evidence showed that the exposure of major vector-borne diseases in dogs in shelters was relatively high in the surveyed areas. Routine detection and control of vector-borne diseases are of paramount importance for reducing the risk of CVBDs transmission in dogs and humans.
\end{abstract}

Keywords: Dirofilariasis, Tick-borne diseases, Infectious diseases, Malaysia.

\section{INTRODUCTION}

Canine vector borne diseases (CVBDs) are increasingly causing public health concern. Their zoonotic potential poses a threat to human health, particularly in the tropics and subtropics as these climatic conditions are conducive for the growth and proliferation of vectors and other reservoir hosts, thus shortening their generation interval (Madder \& Pascucci., 2012; Watanabe et al., 2012). The realm of the vectors, and emergence and spread of CVBDs are the consequences of climate changes, deforestation, habitat changes, and increased relocation of dogs, urbanisation and globalisation (Zell, 2004; Harrus \& Banneth, 2005; Bowman et al., 2009). The increased proximity of human and animal populations due to all the above factors may increase the risk exposure of animals and humans toward these zoonotic pathogens (Otranto et al., 2009; Tziporyet al., 2010; Chomel, 2011).

Domestic dogs may act as reservoir hosts for several species of zoonotic pathogens such as Hepatozoon canis, Ehrlichia canis, Anaplasma platys, Anaplasma phagocytophilum, Bartonella henselae, Borrelia burgdorferi, Dirofilaria repens and Dirofilaria immitis (Otranto et al., 2009).
The clinical signs of these diseases are overlapping. Hepatozoon canis may induce severe clinical manifestations such as lethargy, fever, anorexia, weight loss, lymphadenomegaly, and anemia associated with a high parasite load (Otranto et al., 2009). On the other hand, canine anaplasmosis caused by $A$. phagocytophilum can cause mild to severe acute illness such as anorexia, hyperthermia, vomiting, diarrhea, and neurological signs which is similar to $H$. canis infection (Egenvall et al., 1997). Besides, A. platys targets platelets cell and leads to canine infectious cyclic thrombocytopenia. In addition, canine ehrlichiosis can range from a mild infection to a severe illness leading to death (Harrus \& Banneth, 2005). The diagnosis of canine ehrlichiosis is challenging because of its different phases and multiple clinical-pathological manifestations (Neer et al., 2002; Harrus \& Waner 2011). On the contrary, $D$. immitis the most pathogenic filarid, causes cardiopulmonary dirofilariasis and it is potentially fatal to canids (or canines) (McCall et al., 2008; Rani et al., 2010). Besides, $D$. immitis also infects humans but the infection is incidental and not associated with severe clinical sign. However, the increased cases of human dirofilariasis have been documented in region with high prevalence of canine 
dirofilarial infections. Thus, the detection of heartworm disease and chemoprophylaxis in dogs are important steps for reducing the transmission rate (Lee et al., 2010).

Dogs can be infected with several species of zoonotic pathogens concurrently, depending on the presence and abundancy of the vectors around the area (Nicholson et al., 2010). In Malaysia, the main vectors transmitting CVBDs among dogs include mosquitoes and the brown dog tick Rhipicephalus sanguineus (Vythilingam et al., 2005; Low et al., 2018).

Sheltered dogs have higher chances of being infected with CVBDs as these dogs were unlikely to receive any treatment (Lau et al., 2017). However, the prevalence of CVB pathogens are variable from one another which might be due to the different management protocol in these studied animal shelters. A study conducted in 2016 reported that the seroprevalences of $E$. canis and A. phagocytophilum were $39.5 \%$ and $9.3 \%$, respectively by using serological method (Koh et al., 2015). A recent publication reported that the overall prevalences for E. canis, A. phagocytophilum and D. immitis in three shelters were $55.6 \%, 16.7 \%$ and $10.0 \%$, respectively (Lau et al., 2017). In addition, this study compared the different management protocol of three dog shelters against the prevalence of CVB pathogens and the study suggested that occasional treatment of the dogs might not be effective when compared to scheduled preventive measures (Lau et al., 2017). In the present study, we attempted to understand the relationship between the prevalence of CVB pathogens with different management protocols at two different dog shelters in the state of Selangor, Peninsular Malaysia, and establish their co-infection status using SNAP ${ }^{\circledR} 4 \mathrm{Dx}^{\circledR}$ Plus (IDEXX Laboratories, Westbrook, ME), which can detect the presence of D. immitis antigen, and E. canis, E. ewingii, A. phagocytophilum, $A$. platys and $B$. burgdorferi antibodies.

\section{MATERIALS AND METHODS}

\section{Ethics Statement}

This study was approved by the Medical Ethics Committee of Department Veterinary Services Malaysia (Reference number: JPV: BPI/500-4/1/2 (18)). Written informed consents were received from the owners of the animal shelters.

\section{Sample Collection}

The study was conducted on physically healthy dogs from two different animal shelters (i.e., A and B) located at the state of Selangor, central of Peninsular Malaysia. This study was conducted from the year 2016 to 2017. A total of 103 dog (Shelter A: 51; Shelter B, 52) blood samples (3 ml) were collected by veterinarian in EDTA tubes and stored at $2-4^{\circ}$ prior to analysis from both female and male of different breeds and ages. Based on the physical appearance of the dogs, all dogs were healthy, however no further examination was conducted. The various environmental conditions and exposure status of CVB pathogens observed between Shelter $A$ and Shelter B.

Shelter A is surrounded with forest and river and it is located at rural area which is only accessible via laterite road and muddy road. Due to shortage of funding and lack of volunteers, the shelter does not practice routine preventive measures (i.e., de-tick, shower and preventive medicine for heartworm disease). The shelter is divided into two types of confinement system whereby some of the dogs were confined in smaller groups whereas approximately 200 dogs were left to roam freely in a big compound (approximately 6 metres $x$
5 metres). Dogs confined in smaller groups were puppies, sick dogs and dogs that could not socialise well with other dogs. Some of these dogs were severely infested with ticks and fleas. During the blood collection, ticks samples were also collected and screened for various pathogens (Low et al., 2017).

On the other hand, dogs in Shelter B were better maintained, confined in different groups in the kennels with concrete floor. All dogs were allowed to run freely during the fixed schedules in the compound of the animal shelter surrounded by vegetation. Dog kennels were cleaned thrice daily. All dogs were given a bath weekly, and using anti tick shampoo as routine preventive measures to prevent infestation of ticks and fleas. However, heartworm prevention was not administered due to the high cost of the medications. None of the dogs were found infected with ticks and fleas during blood collection. All the dogs in both Shelter A and Shelter B were fed twice a day. All the dogs in the shelter were physically fit and healthy.

\section{Serologic Test}

A serological diagnostic test was performed by using a commercial lateral flow assay, (SNAP 4Dx test kits IDEXX Laboratories, Westbrook, ME) in accordance with the manufacturer's protocol. This kit can detect antibodies against $A$. phagocytophilum/A. platys, E. canis/E. ewingii, $B$. burgdorferi and antigen of $D$. immitis. The seropositive values of tick-borne pathogens (Anaplasma spp., Ehrlichia spp., B. burgdorferi) reported in the present study were interpreted as current infection with/or previous exposure to the pathogens. The test results collected were compiled accordingly and all seroprevalence of the pathogens were calculated and expressed in percentage. The 95\% confidence interval of all estimates was calculated.

\section{RESULTS}

Exposure to at least one vector-borne pathogen was documented in 63 (61.2\%) dogs (Table 1). Out of 103 dogs blood samples, antibodies of Ehrlichia spp. and Anaplasma spp. were detected in 46/103 (44.7\%) and 31/103 (30.1\%) of dogs, respectively. On the other hand, antigen of $D$. immitis was detected in $14 / 103(13.6 \%)$ of dogs. However, antibodies of $B$. burgdorferi were not detected in this study. A total of 19 (18.5\%) dogs were tested seropositive for Anaplasma spp. + Ehrlichia spp., two (1.9\%) dogs seropositive for Anaplasma spp. $+D$. immitis and one (1.0\%) dog seropositive with D. immitis + Ehrlichia spp. Interestingly, three (2.9\%) dogs were tested seropositive with triple infections (Anaplasma spp. + Ehrlichia spp. + D. immitis).

In shelter A, 26 out of 52 dogs (51\%), which represent half of the population were seropositive for Ehrlichia spp. and 22 dogs (43.1\%) were seropositive for Anaplasma spp. Four dogs $(7.9 \%)$ were infected with $D$. immitis. Mixed infection of Ehrlichia spp., Anaplasma spp. and D. immitis (double infection and triple infection) was observed in 15 dogs (29.4\%).

On the other hand, in shelter B, out of 53 dogs, 20 (38.5\%) dogs and nine (17.3\%) dogs were seropositive for Ehrlichia spp. and Anaplasma spp., respectively. However, the infection of $D$. immitis was higher in shelter $B$, in which nine dogs were infected (19.23\%). Eleven dogs (21.2\%) were observed to have mixed infection of CVBDs (double exposure and triple exposure) in this shelter. None of the dogs were found seropositive with $B$. burgdorferi for both shelters. 
Table 1. Exposure status of D. immitis, Anaplasma spp., B. burgdorferi and Ehrlichia spp. in 103 dogs in Selangor, Malaysia

\begin{tabular}{|c|c|c|c|c|c|c|c|c|c|}
\hline \multirow{2}{*}{ Pathogens } & \multicolumn{3}{|c|}{ Shelter $A(N=51)$} & \multicolumn{3}{|c|}{ Shelter $B(N=52)$} & \multicolumn{3}{|c|}{ Total $(\mathrm{N}=103)$} \\
\hline & $\mathrm{N}$ & $\%$ & Cl (95\%) & $n$ & $\%$ & $\mathrm{Cl}(95 \%)$ & $\mathrm{n}$ & $\%$ & $\mathrm{Cl}(95 \%)$ \\
\hline \multicolumn{10}{|l|}{ Overall } \\
\hline D. immitis & 4 & 7.84 & $2.2-18.9$ & 10 & 19.23 & $9.0-32.0$ & 14 & 13.60 & $7.6-21.8$ \\
\hline Ehrlichia spp. & 26 & 50.98 & $36.6-65.2$ & 20 & 38.46 & $24.8-52.1$ & 46 & 44.66 & $34.9-54.8$ \\
\hline Anaplasma spp. & 22 & 43.14 & $29.3-57.8$ & 9 & 17.31 & $8.1-29.8$ & 31 & 30.10 & $21.4-39.9$ \\
\hline \multicolumn{10}{|l|}{ Single infection } \\
\hline D. immitis & 1 & 1.96 & $0.0-10.4$ & 7 & 13.46 & $5.6-25.8$ & 8 & 7.77 & $3.4-14.7$ \\
\hline Ehrlichia spp. & 10 & 19.61 & $9.8-33.1$ & 12 & 23.08 & $12.5-36.8$ & 23 & 22.3 & $14.7-31.6$ \\
\hline Anaplasma spp. & 8 & 15.69 & $7.0-28.6$ & 0 & 0.00 & - & 7 & 6.80 & $2.8-13.5$ \\
\hline \multicolumn{10}{|l|}{ Double infection } \\
\hline Anaplasma spp. + Ehrlichia spp. & 12 & 23.53 & $12.8-37.5$ & 7 & 13.46 & $5.6-25.8$ & 19 & 18.45 & $11.5-27.3$ \\
\hline Anaplasma spp. + D.immitis & 1 & 1.96 & $0.0-10.4$ & 1 & 1.92 & $0.0-10.3$ & 2 & 1.94 & $0.2-6.8$ \\
\hline D. immitis + Ehrlichia spp. & 1 & 1.96 & $0.0-10.4$ & 0 & 0.00 & - & 1 & 0.97 & $0.0-5.3$ \\
\hline \multicolumn{10}{|l|}{ Triple infection } \\
\hline Anaplasma spp. + Ehrlichia spp. $+D$. immitis & 1 & 1.96 & $0.0-10.4$ & 2 & 3.85 & $0.5-13.2$ & 3 & 2.91 & $0.6-8.3$ \\
\hline
\end{tabular}

\section{DISCUSSION}

Of 103 dogs examined, 46 (44.7\%) and 31 (30.1\%) dogs were tested seropositive for Ehrlichia spp. and Anaplasma spp., respectively. The results obtained from the present study were comparable with previous studies conducted in Malaysia. A recent study reported higher seroprevalence of Ehrlichia spp. (55.6\%), and lower seroprevalences of Anaplasma spp. $(16.7 \%)$ and D. immitis $(10.0 \%)$ as compared to our study (Anaplasma spp. $=30.10 \%$; D. immitis $=13.6 \%$ ) (Lau et al., 2017). In another study, the seroprevalences of Ehrlichia spp. (39.5\%) and Anaplasma spp. (9.3\%) were relatively lower (Koh et al., 2015).

Molecular identification of these pathogens was performed on the blood samples in shelter $A$ in a recent study (Low et al., 2018). DNAs of $A$. platys (8/51=15.67\%) and E. canis $(31 / 51=60.78 \%)$ were detected, confirming that $A$. platys and $E$. canis were the causative agents for the tick-borne diseases in this study area.

While there was a moderate agreement (62.50\%) between the molecular assay and SNAP 4Dx results for $E$. canis, the results for $A$. platys were less satisfactory $(36.40 \%)$. These results were expected because antibody test cannot distinguish current infection or previous exposure to the pathogens. In contrast, molecular detection can provide high sensitivity and specificity results for current infection of the pathogens. Thus, it is suggested that the combined use of PCR and serological assays could heighten the detection of infection with or exposure to CVBDs effectively. Additionally, eights dogs and five dogs were detected positive with the DNA of Hepatozoon canis (15.7\%) and Babesia vogeli (9.8\%) respectively, in shelter A (Prakash et al., 2018a, 2018b).

Borrelia burgdorferi is a tick-borne spirochete that causes Lyme diseases in many animals including dogs, horses, cattle and human (Joppert et al., 2001; Vorou et al., 2007). It can cause severe arthritis or, rarely, glomerulonephritis in dogs, although some dogs can be asymptomatic (Herrin et al., 2018). Borrelia burgdorferi in dog was not found in the present and previous studies in Malaysia (Koh et al., 2015). Nevertheless, the presence of antibodies against this bacterium was found in human blood donors and patients in Malaysia (Tay et al., 2002).
An earlier study conducted in southern Peninsular Malaysia reported a very low seroprevalence of $D$. immitis (1.33\%) (Ng et al., 2012), possibly because of majority of the samples were pet dogs. Thus, exposures of these pet dogs to the infected mosquitoes were lower as compared to the shelter dogs from our study. Likewise, similar results were also reported in owned dogs. In 1993, 10\% of owned dogs were detected positive with $D$. immitis based on microscopic analysis (Dhaliwal \& Sani, 1993.). By contrast, prevalence of D. immitis in shelter dogs ranged from 26 to $42 \%$, based on Knott's Concentration Test (KCT) and necropsy technique (Mulim, 1970; Retnasabapathy \& Khoo, 1976; Toh, 2002).

Similar studies were also conducted in other countries in Southeast Asia. A recent study in Thailand reported lower seroprevalences of Anaplasma spp. (29.4\%) and Ehrlichia spp. (25.0\%) (Piratae et al., 2019) while the seroprevalences of Anaplasma spp. (47.1\%), Ehrlichia spp. (22.51\%), D. immitis (17.8\%) reported in Northeast India (Borthakur et al., 2015) were higher compared with the results observed in our study. Result variation and inconsistency in different countries can be due to different target population either from owned dogs or sheltered dogs, and different geographical and ecological variations (Jung et al., 2012).

Co-infection of pathogens may complicate the interpretation of the clinical manifestations typically associated with single vector-borne disease. This can occur simultaneously or a sequential exposure to several infected vectors, or by a single vector that was infected with multiple pathogens (Kordick et al., 1999; Beall et al., 2008; Gaunt et al., 2010). Overall, the co-infection rates $(24.27 \%)$ were higher compared to a previous study (Koh et al., 2015). The co-infection of several pathogens can lead to severe clinical manifestations. The complicated clinical manifestations which are similar to other diseases may complicate diagnosis and veterinarians often mistakenly linked other diseases to CVBDs (DantasTorres, 2008; Beugnet \& Marie, 2009; Menn et al., 2010). In the current study, the total seroprevalence of Shelter A (66.7\%) was higher than Shelter B (55.8\%). The seroprevalences of Anaplasma spp. and Ehrlichia spp. were found to be higher at Shelter A (Anaplasma spp.: 43.1\%, Ehrlichia spp.: 51.0\%) than Shelter B (Anaplasma spp.: 17.3\%, Ehrlichia spp.: 38.5\%). The most likely explanation is the fact that the dogs in Shelter $B$ 
were bathed every week and routine preventive measures were done to prevent infestation of ticks and fleas. This decreases the infestation of ticks which act as vectors in transmitting both pathogens from one dog to another. All dogs in Shelter A were hardly bathed due to the lack of manpower. Indeed, most of the animal shelters in Malaysia were overcrowded with dogs and operated with insufficient or limited funding, inexperienced staffs and expertise in managing the infections and diseases. All these limitations resulted in different management protocols of CVBDs for the animal shelters. Management varies depending on their socioeconomic status, expertise and manpower (Vorou et al., 2007; Lau et al., 2018). On the other hand, the seroprevalence of $D$. immitis in Shelter B (19.23\%) was higher than Shelter A (7.84\%). Unfortunately, mosquito surveillance was not conducted on both sites to determine the vector that may contribute to the infection of $D$. immitis. However, Aedes albopictus which has been discriminated as a vector for D. immitis (Cancrini et al., 2003) could be found in the study sites. Proper mosquito control needs to be done to reduce the risk of infection.

In the nutshell, the current study showed that $61.2 \%$ $(63 / 103)$ of the dogs were seropositive with at least one pathogen. The prevalence was alarmingly high, suggesting that the exposure to CVBDs was ubiquitous, especially among dogs in the animal shelters. Limited veterinary care and financial constraints in animal shelters were other compounding factors. As a result, most of the animal shelters in Malaysia are not able to provide heartworm and tick prevention periodically.

This study provides a better understanding on exposure of CVBDs infecting sheltered dogs in Selangor, Malaysia which may serve as baseline information for future investigations and preventive action in order to protect dogs and control the risk of transmission of CVBDs to human and other animals. Further investigations involving a combined dog and human tick-borne disease surveillance data could enhance both public health and animal health. Surveillance studies on other vector-borne pathogens also need to be conducted to get a better understanding of the current status of CVBD transmission in Malaysia.

\section{ACKNOWLEDGEMENTS}

This study was financially supported by the University of Malaya research grant (PG098-2016A) and the Higher Institution Centre of Excellence (HICOE) program (MO0022019). We would like to express our gratitude to the owners of the animal shelters and veterinarians for their assistance in sample collection.

\section{Conflict of interest statement}

No potential conflicts of interests with respect to authorship and/ or publication of this article.

\section{REFERENCES}

Beall, M.J., Chandrashekar, R., Eberts, M.D., Cyr, K.E., Diniz, P.P., Mainville, C., Hegarty, B.C., Crawford, J.M. \& Breitschwerdt, E.B. (2008). Serological and molecular prevalence of Borrelia burgdorferi, Anaplasma phagocytophilum, and Ehrlichia species in dogs from Minnesota. Vector Borne Zoonotic Diseases 8(4): 455-464. DOI: 10.1089/vbz.2007.0236

Beugnet, F. \& Marie, J.L. (2009). Emerging arthropod-borne diseases of companion animals in Europe. Veterinary Parasitology 163(4): 298-305. https://doi.org/10.1016/ j.vetpar.2009.03.028
Borthakur, S.K., Deka, D.K., Islam, S., Sarma, D.K. \& Sarmah, P.C. (2015). Prevalence and molecular epidemiological data on Dirofilaria immitis in dogs from northeastern states of India. The Scientific World Journal 2015: 1-7. https://doi.org/ $10.1155 / 2015 / 265385$

Bowman, D., Little, S.E., Lorentzen, L., Shields, J., Sullivan, M.P. \& Carlin, E.P. (2009). Prevalence and geographic distribution of Dirofilaria immitis, Borrelia burgdorferi, Ehrlichia canis and Anaplasma phagocytophilum in dogs in the United States: results of a national clinic-based serologic survey. Veterinary Parasitolgy 160: 138-148. https://doi.org/10.1016/j.vetpar.2008.10.093

Cancrini, G., Frangipani di Regalbono, A., Ricci, I., Tessarin, C., Gabrielli, S. \& Pietrobelli, M. (2003). Aedes albopictus in a natural vector of Dirofilaria immitis in Italy. Veterinary Parasitology 118(3-4): 195-202. https://doi.org/10.1016/ j.vetpar.2003.10.011

Chomel, B. (2011). Tick-borne infections in dogs - an emerging infectious threat. Veterinary Parasitolgy 179(4): 294-301. https://doi.org/10.1016/j.vetpar.2011.03.040

Dantas-Torres, F. (2008). Canine vector-borne diseases in Brazil. Parasites \& Vectors 1(1): 1. https://doi.org/10.1186/ 1756-3305-1-25

Dhaliwal, G.K. \& Sani, R. (1993). The prevalence of canine dirofilariasis in Kuala Lumpur and host risk factors. Tropical Biomedicine 10(1): 73-76.

Egenvall, A.E., Hedhammar, A.A. \& Bjoersdorff, A.I. (1997). Clinical features and serology of 14 dogs affected by granulocytic ehrlichiosis in Sweden. Veterinary Record 140: 222-226. https://doi.org/10.1136/vr.140.9.222

Gaunt, S.D., Beall, M.J., Stillman, B.A., Lorentzen, L., Diniz, P.P.V.P., Chandrashekar, R. \& Breitschwerdt, E.B. (2010). Experimental infection and co-infection of dogs with Anaplasma platys and Ehrlichia canis: hematologic, serologic and molecular findings. Parasites \& Vectors 3(33): 33-43. https://doi.org/10.1186/1756-3305-3-33

Harrus, S. \& Baneth, G. (2005). Drivers for the emergence and reemergence of vector-borne protozoal and bacterial diseases. International Journal of Parasitology 35: 1309-1318. https://doi.org/10.1016/j.ijpara.2005.06.005

Harrus, S. \& Waner, T. (2011). Diagnosis of canine monocytotropic ehrlichiosis (Ehrlichia canis): An overview. The Veterinary Journal 187(3): 292-296. https://doi.org/10.1016/ j.tvjl.2010.02.001

Herrin, B.H., Beall, M.J., Feng, X., Papeş, M. \& Little, S.E. (2018). Canine and human infection with Borrelia burgdorferi in the New York City metropolitan area. Parasites \& Vectors 11(1): 1-10. https://doi.org/10.1186/s13071-018-2774-z

Joppert, A.M., Hagiwara, M.K. \& Yoshinari, N.H. (2001). Borrelia burgdorferi antibodies in dogs from Crotia country, São Paulo State, Brazil. The Revista do Instituto de Medicina Tropical de São Paulo 43(5): 251-255. http://dx.doi.org/ 10.1590/S0036-46652001000500003

Jung, B.Y., Gebeyehu, E.B., Seo, M.G., Byun, J.W., Kim, H.Y. \& Kwak, D. (2012). Prevalence of vector-borne diseases in shelter dogs in Korea. Veterinary Record 171: 249. http://dx.doi.org/10.1136/vr.100650

Koh, F.X., Panchadcharam, C. \& Tay, S.T. (2015). Vector-borne diseases in stray dogs in Peninsular Malaysia and molecular detection of Anaplasma and Ehrlichia spp. from Rhipicephalus sanguineus (Acari: Ixodidae) ticks. Journal of Medical Entomology 53(1): 183-187. https://doi.org/10.1093/ jme/tjv153

Kordick, S.K., Breitschwerdt, E.B., Hegarty, B.C., Southwick, K.L., Colitz, C.M., Hancock, S.I., Bradley, J.M., Rumbough, R., Mcpherson, J.T. \& MacCormack, J.N. (1999). Coinfection with multiple tick borne pathogens in a Walker Hound kennel 
in North Carolina. Journal of Clinical Microbiology 37(8): 26312638. https://doi.org/10.1128/JCM.37.8.2631-2638.1999

Lee, A.C.Y., Montgomery, S.P., Theis, J.H., Blagburn, B.L. \& Eberhard, M.L. (2010). Public health issues concerning the widespread distribution of canine heartworm disease. Trends in Parasitology 26: 168-73. https://doi.org/10.1016/ j.pt.2010.01.003

Lau, S.F., Dolah, R.N., Mohammad, K. \& Abdul-Rani, P.A.M. (2017). Canine vector borne diseases of zoonotic concern in three dog shelters in Peninsular Malaysia: The importance of preventive measures. Tropical Biomedicine 34(1): 72-79.

Low, V.L., Chen, C.D., Lee, H.L., Lim, P.E., Leong, C.S. \& SofianAzirun, M. (2012). Nationwide distribution of Culex mosquitoes and associated habitat characteristics at residential areas in Malaysia. Journal of American Mosquito Control Association 28: 160-169. https://doi.org/10.2987/126235R.1

Low, V.L., Prakash, B.K., Tan, T.K., Sofian-Azirun, M., Anwar, F.H.K., Vinnie-Siow, W.Y. \& AbuBakar, S. (2017). Pathogens in ectoparasites from free-ranging animals: Infection with Rickettsia asembonensis in ticks, and a potentially new species of Dipylidium in fleas and lice. Veterinary Parasitology 245: 102-105. https://doi.org/10.1016/j. vetpar.2017.08.015

Low, V.L., Prakash, B.K., Lim, Y.A.L., Tan, T.K., Vinnie-Siow, W.Y., Sofian-Azirun, M. \& Abubakar, S. (2018). Detection of Anaplasmataceae agents and coinfection with other tickborne protozoa in dogs and Rhipicephalus sanguineus sensu lato ticks. Experimental and Applied Acarology 75(4): 429-435. https://doi.org/10.1007/s10493-018-0280-9

Madder, M. \& Pascucci, I. (2012). Ticks and ticks-borne diseases: Geographical distributions and control strategies in the Euro-Asian region. CABI Agriculture and Bioscience Journal 3: 27-32.

McCall, J.W., Genchi, C., Kramer, L.H., Guerrero, J. \& Venco, L. (2008). Heartworm disease in animals and humans. Advance in Parasitology 66: 193-285. https://doi.org/10.1016/ S0065-308X(08)00204-2

Menn, B., Lorentz, S. \& Naucke, T.J. (2010). Imported and travelling dogs as carriers of canine vector-borne pathogens in Germany. Parasites \& Vectors 3(34): 1-7. https:// doi.org/10.1186/1756-3305-3-34

Mullin, S.W. (1970). Canine filariasis in Kuala Lumpur: prevalence and diagnosis. The Malayan Veterinary Journal 1: 11-13.

Neer, T.M., Breitschwerdt, E.B., Greene, R.T. \& Lappin, M.R. (2002). Consensus statement on ehrlichial disease of small animals from the infectious disease study group of the ACVIM. Journal of Veterinary Internal Medicine 16: 309-315. https://doi.org/10.1892/0891-6640(2002)016<0309: csoedo $>2.3 . c 0 ; 2$

Nicholson, W.L., Allen, K.E., McQuiston, J.H., Breitschwerdt, E.B. \& Little, S.E. (2010). The increasing recognition of rickettsial pathogens in dogs and people. Trends in Parasitology 26(4): 205-212. https://doi.org/10.1016/j.pt. 2010.01.007

Ng, K.L., Lee, E.L. \& Sani, R.A. (2012). Low prevalence of Dirofilaria immitis in dogs in Johor Bahru, Malaysia as a reflection of vector availability? (2012) Tropical Biomedicine 29(1): 187190.

Otranto, D., Dantas-Torres, F. \& Breitschwerdt, E.B. (2009). Managing canine vector borne diseases of zoonotic concern: part one. Trends in Parasitology 25(4): 157-163. https://doi.org/10.1016/j.pt.2009.01.003
Otranto, D., Dantas-Torres, F., Weighl, S., Latrofa, M.S., Stannek, D., Decaprariis, D., Capelli, G. \& Baneth, G. (2011). Diagnosis of Hepatozoon canis in young dogs by cytology and PCR. Parasites \& Vector 4(55). https://doi.org/10.1186/ 1756-3305-4-55

Piratae, S., Senawong, P., Chalermchat, P., Harnarsa, W. \& Saechue, B. (2019). Molecular evidence of Ehrlichia canis and Anaplasma platys and the association of infections with hematological responses in naturally infected dogs in Kalasin, Thailand. Veterinary World 12(1): 131-135. https:// doi.org/10.14202/vetworld.2019.131-135

Prakash, B.K., Low, V.L., Tan, T.K., Vinnie-Siow, W.Y., Lim, Y.A.L., Morvarid, A.R., Azman, A.S., Yeong, Y.S., AbuBakar, S. \& Sofian-Azirun, M. (2018). Detection of Hepatozoon canis in the brown dog tick Rhipicephalus sanguineus and domestic dogs in Peninsular Malaysia. Journal of Medical Entomology 55: 1346-1348. https://doi.org/10.1093/jme/tjy081

Prakash, B.K., Low, V.L., Vinnie-Siow, W.Y., Tan, T.K., Lim, Y.A.L., Morvarid, A.R., AbuBakar, S. \& Sofian-Azirun, M. (2018). Detection of Babesia spp. in dogs and their ticks from Peninsular Malaysia: Emphasis on Babesia gibsoni and Babesia vogeli infections in Rhipicephalus sanguineus sensu lato (Acari: Ixodidae). Journal of Medical Entomology 55: 1337-1340. https://doi.org/10.1093/jme/tjy072

Rani, M.A., Irwin, P.J., Gatne, M., Coleman, G.T., McInnes, L.M. \& Traub, R.J. (2010). A survey of canine filarial diseases of veterinary and public health significance in India. Parasites \& Vectors 3(1): 1. https://doi.org/10.1186/1756-3305-3-30

Retnasabapathy, A. \& Khoo, T.S. (1976). Incidence of canine heartworm (Dirofilaria immitis) in Malaysia. Veterinary Record 98: 68-69. https://doi.org/10.1136/vr.98.4.68

Tay, S.T., Kamalanathan, M. \& Rohani, M.Y. (2002). Borrelia burgdorferi (strain B. afzelii) antibodies among Malaysian blood donors and patients. Southeast Asian Journal of Tropical Medical and Public Health 33(4): 787-793.

Toh, P.Y. (2002). Blood parasites in local dogs in Selangor. Degree in Veterinary Medicine Thesis. Faculty of Veterinary Medicine. Universiti Putra Malaysia.

Tzipory, N., Crawford, P.C. \& Levy, J.K. (2010). Prevalence of Dirofilaria immitis, Ehrlichia canis and Borrelia burgdorferi in pet dogs, racing greyhounds, and shelter dogs in Florida. Veterinary Parasitology 171(1): 136-139. https://doi.org/ 10.1016/j.vetpar.2010.03.016

Vorou, R.M., Papavassiliou, V.G. \& Tsiodras, S. (2007). Emerging zoonoses and vector-borne infections affecting humans in Europe. Epidemiology and Infection 135(08): 1231-1247. https://doi.org/10.1017/\$0950268807008527

Vythilingam, I., Mooto, P., Jeffery, J., Parameswaran, M.S., Lee, C. \& Robinson, W. (2005). Potential mosquito (Diptera: Culicidae) vectors of Dirofilaria immitis (Filariidae: Onchocercidae) in two urban areas of Kuala Lumpur and its prevalence in stray dogs. International Conference on Urban Pests (ICUP).

Watanabe, M. (2012). Main pet arthropod-borne diseases in Asia. $9^{\text {th }}$ Merial symposium on parasitosis and arthropodborne diseases. Tokyo $6^{\text {th }}-9^{\text {th }}$ November 2012, 18-21.

Zell, R. (2004). Global climate change and the emergence/ re-emergence of infectious diseases. International Journal of Medical Microbiology 293(37): 16-26. https://doi.org/ 10.1016/s1433-1128(04)80005-6 